\title{
A plasmonic hybrid nanostructure with controlled interaction strength
}

\author{
Justyna Grzelak, ${ }^{1}$ Bartosz Krajnik, ${ }^{1}$ Mark D. Thoreson, ${ }^{2,3}$ Piotr Nyga, ${ }^{2,3,4}$ Vladimir M. Shalaev, ${ }^{2,3}$ \\ and Sebastian Mackowski ${ }^{*}$ \\ ${ }^{1}$ Optics of Hybrid Nanostructures Group, Institute of Physics, Faculty of Physics, Astronomy, and Informatics, \\ Nicolaus Copernicus University, Grudziadzka 5/7, 87-100 Toruń, Polska \\ ${ }^{2}$ School of Electrical and Computer Engineering, Purdue University, West Lafayette, Indiana 47907, USA \\ ${ }_{3}^{3}$ Birck Nanotechnology Center, Purdue University, West Lafayette, Indiana 47907, USA \\ ${ }^{4}$ Institute of Optoelectronics, Military University of Technology, \\ Kaliskiego 2, 00-908 Warszawa, Polska
}

Received May 16, 2013; accepted June 23, 2013; published June 30, 2013

\begin{abstract}
We describe a novel plasmonic hybrid nanostructure based on a silver island film covered with a dielectric silica layer. The thickness of the silica layer is varied from 0 to approximately $46 \mathrm{~nm}$ on a single sample, thus allowing for continuous variation of the interaction strength between plasmon excitations in the metallic film and the excited states of pigments comprising photosynthetic complexes used to probe this interaction. While the largest separation between the silver film and photosynthetic complexes provides fluorescence featuring mono-exponential decay, thinner silica spacer distances show bi-exponential decay. The intensity of the fast component, which is attributed to the emission of photosynthetic complexes coupled to plasmon excitations, strongly decreases as a function of the spacer thickness. The interaction is stronger for excitation wavelengths resonant with plasmon absorption in the metallic layer.
\end{abstract}

The interaction between plasmon excitations in metallic nanoparticles and fluorophores depends strongly upon the distance between these two nanostructures [1]. Typically, for distances larger than $40-50 \mathrm{~nm}$, the interaction is very weak and leads to no measureable changes in the absorption or emission of fluorophores. When approaching a metallic nanoparticle, however, and reaching a distance between $10 \mathrm{~nm}$ and $25 \mathrm{~nm}$, the emission intensity of fluorescence shows a gradual increase. This enhancement results from an increase in fluorophore absorption and/or emission rate due to the local influence of plasmon excitations in metallic nanoparticles. Lastly, for separations comparable or less than $5 \mathrm{~nm}$, the emission exhibits quenching attributed to non-radiative energy transfer from a fluorophore to a metallic nanoparticle. Various nanostructures have been investigated from the point of view of plasmonic effects, including organic molecules $[1,2]$, semiconductor

\footnotetext{
*E-mail: mackowski@fizyka.umk.pl
}

nanocrystals [3], conjugated polymers [4], and multichromophoric photosynthetic complexes $[5,6]$.

Due to the strong sensitivity of fluorescence enhancement to the distance separating a metallic nanoparticle from a fluorophore, several strategies have been proposed to control and study this important facet of plasmonic enhancement. Metallic nanoparticles were covered with dielectric layers or functionalized with active groups that enable robust conjugation with fluorophores, and thin polymer layers have been applied in previous efforts. However, all these methods inhibit the fabrication of a structure in which the distance between the fluorophore and the metallic nanoparticle can be gradually varied, thus allowing the study of the distance dependence of the interaction strength. Perhaps the only realization of such a system concerns the metallic nanoparticle attached to an ultrasmall tip that can be moved with high precision in order to vary the distance from the nanoparticle to a molecule [1]. This method is highly advanced and quite limited in regard to any practical applications.

In this contribution, we describe the results of fluorescence spectroscopy experiments carried out on a plasmonic hybrid nanostructure composed of a silver island film and a light-harvesting complex, peridininchlorophyll-protein (PCP). The metallic substrate was fabricated on a single glass microscope slide in such a way that the thickness of a silica spacer covering the metallic layer was varied continuously from 0 to $46 \mathrm{~nm}$. The transmission of the metallic layer covered with the silica spacer shows essentially no variation across the substrate, indicating that the metallic nanoparticle film is unperturbed by the varying silica thickness. The evaluation of the results of time-resolved fluorescence spectroscopy indicates that the strength of the interaction between plasmon excitations in the silver film and the excited states in the PCP complexes feature a clear, 
gradual increase with the reduction of the silica layer thickness.

The structure studied in this work was fabricated using electron-beam-assisted evaporation on a glass microscope slide with dimensions of $25 \mathrm{~mm}$ by $75 \mathrm{~mm}$. The fabricated structure consists of a silver island film, also called a semicontinuous silver film, evaporated over the whole surface of a microscope slide (Fig. 1b). The nominal thickness of the film, measured during the fabrication with a quartz oscillator monitoring system, was $8.3 \mathrm{~nm}$. The general procedure of metallic layer evaporation has been described in detail previously [7, 8]. In the next step, the metallic layer was covered with a silica layer in the same evaporation process (i.e., without opening the vacuum evaporation chamber). Importantly, the thickness of this layer was varied continuously along the length of a microscope slide. The gradient in $\mathrm{SiO}_{2}$ layer thickness was achieved by the use of a shutter attached to a motorized linear stage moving with a constant speed during the deposition of the silica material [9]. Prior to the $\mathrm{SiO}_{2}$ layer evaporation, the shutter was positioned to screen the sample from the $\mathrm{SiO}_{2}$ vapour. During evaporation, the shutter was moved at a constant rate to expose more and more of the microscope slide to the silica vapour. A gradient of silica thickness was therefore achieved with the thickest $\mathrm{SiO}_{2}$ layer occurring where the slide was exposed to the vapour flux for the longest time. A 5-mm-wide stripe on one of the microscope slides was left uncovered with $\mathrm{SiO}_{2}$ as a reference region. The transmission spectra of the obtained substrate were measured in the visible and near-infrared wavelength ranges using a V-VASE ellipsometer (J. A. Woollam Co.) at various locations on the sample corresponding to different silica thicknesses. Examples of the transmission spectra obtained for several locations along the sample are shown in Fig. 1a. The strong plasmonic character of the substrate is evident through a resonance band that appears at around $600 \mathrm{~nm}$; the resonance is fairly broad, but its position and overall shape are independent of the position along the substrate (black, red and green spots on the sample surface in Fig. 1b). This indicates high uniformity of the metallic nanoparticle film structure and no dependence on the silica layer thickness. On such a prepared substrate we deposit a solution of the PCP complexes by drop-casting the solution at defined locations. The size of these droplets was less than $2 \mathrm{~mm}$ in diameter, as schematically displayed in Fig. 1b.

Fluorescence transients of light-harvesting complexes coupled to the nanostructured metallic film were imaged using a confocal fluorescence microscope based on Olympus infinity-corrected microscope objective LMPlan $50 \mathrm{x}$ with a numerical aperture of 0.5 and a working distance of $6 \mathrm{~mm}$ [10]. For excitation, two lasers with wavelengths of 405 and 640nm were used, each providing a pulsed output with 30 -ps pulses and a repetition rate of $80 \mathrm{MHz}$. Detection was performed with a fast avalanche photodiode (idQuantique). Emission of the PCP complexes was extracted using a band-pass filter HQ 670/10 (Chroma).

a)

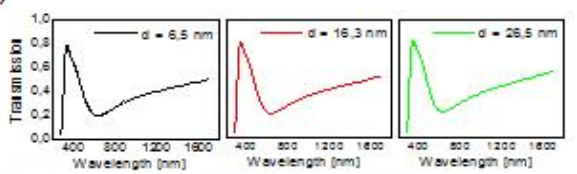

b)

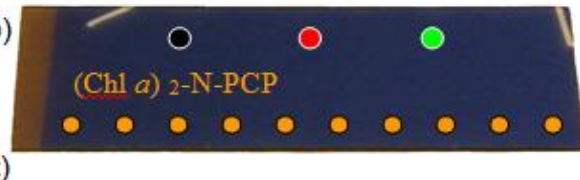

c)

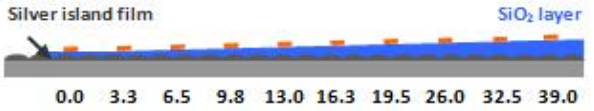

d)
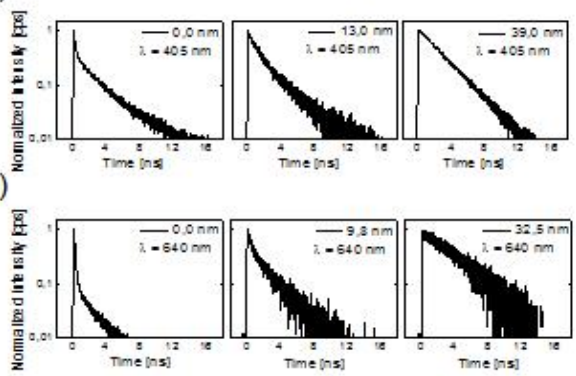

Fig. 1. Image of the structure studied in this work with positions (black, red, and green spots), from which transmission spectra shown in the upper row were taken. Below the image are examples of normalized fluorescence transients collected out of three locations across the sample for both $405 \mathrm{~nm}$ and $640 \mathrm{~nm}$ excitations.

In Fig. 1d and Fig. 1e we present the examples of normalized fluorescence lifetime measurements obtained for several locations across the substrate, and thus for different thicknesses of the silica layer. The data was obtained with excitation at $405 \mathrm{~nm}$ and 640nm, respectively. While the first wavelength is far from the plasmon resonance in the silver island film, the second excitation wavelength $(640 \mathrm{~nm})$ matches the resonance rather well. In both cases the transients measured for the spacer thickness larger than $30 \mathrm{~nm}$ exhibit monoexponential behaviour with a decay constant similar to the reference PCP complexes on a plain glass substrate. On reducing the thickness of the silica spacer transients start to develop a second, faster component whose intensity increases with decreasing spacer thickness. Consequently, for spacers thinner than $15 \mathrm{~nm}$, the fluorescence decay is bi-exponential: the fast component $\left(\mathrm{t}_{1}\right.$, black points in Fig. 2) originates from the PCP complexes interacting with plasmon excitations in the metallic layer, while the slower 
component ( $t_{2}$, red points in Fig. 2) can be tentatively attributed to the PCP complexes that are far enough from the metallic layer so that their optical properties remain unaffected by its presence. We note that the transients collected for the same droplet on the sample surface are virtually identical to each other, with the exception of intensity, which varies, to some degree, across every spot. Furthermore, the intensity of the fast component measured for the sample excited with $405 \mathrm{~nm}$ is significantly smaller as compared with the excitation at $640 \mathrm{~nm}$, which is more strongly resonant with the plasmon maximum of the silver island film.
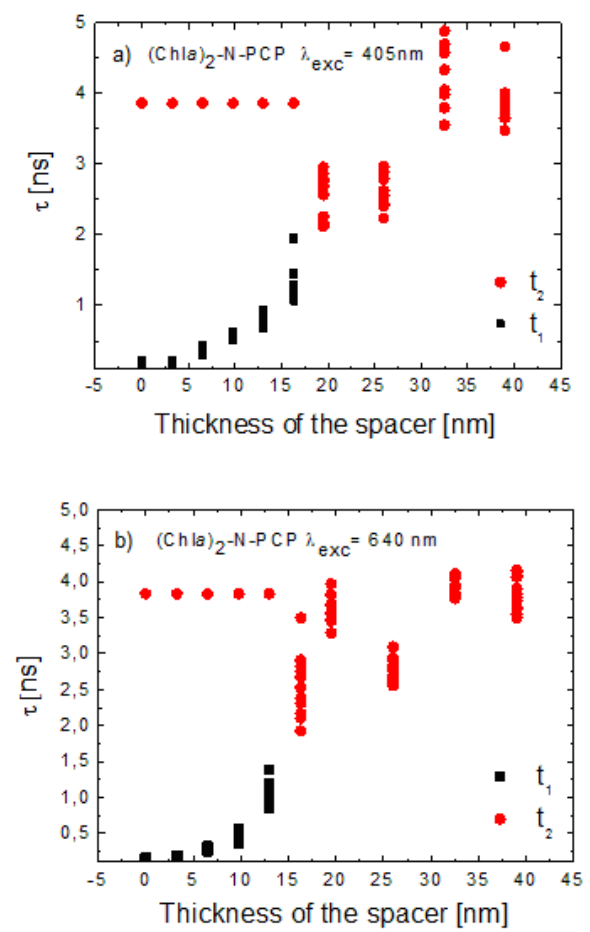

Fig. 2. Fluorescence decay times extracted from transients measured for spots along the plasmonic substrate. For a detailed description of the fitting procedure - see text.

In order to quantify the distance dependence of the optical properties of the PCP complexes coupled to a silver island film, we analysed the transients by fitting either one or two exponential decay constants. The result of the analysis is displayed in Fig. 2. In the case of relatively thick spacer thicknesses, we used a monoexponential decay and fit all of the transients measured for the droplet. As can be seen in Fig. 2, for the largest spacers the fluorescence decay is constant, with some variation between transients measured for a given droplet. In the intermediate range of spacer thickness (around 20$25 \mathrm{~nm}$ ), the decay lifetime becomes shorter, however, the fits are still of good quality. For the transients that exhibit clear bi-exponential behaviour (for spacers around 15-17 $\mathrm{nm}$ and below), we used two decay constants for fitting with one parameter fixed at $3.8 \mathrm{~ns}$, which corresponds roughly to the reference decay of the PCP complexes on a bare glass substrate. In this way, we are able to extract information on the spacer-thickness dependence for both the decay time and the intensity.

The results displayed in Fig. 2 indicate that with a decreasing thickness in the silica spacer, the decay time is strongly reduced. This reduction is accompanied with an increase in the intensity associated with this decay constant. The reduction of the decay time suggests a strong modification of the radiative properties of the PCP complexes upon coupling with plasmonic excitations in the silver island film. The increase of the intensity contribution associated with the fast decay constant implies that the relative amount of the PCP complexes interacting with the metallic nanostructure increases for thinner spacers. The change of the decay constant proves that the fabricated silver island film covered with a silica spacer of varying thickness can be used for detailed evaluations of plasmonic interactions with arbitrary emitters in a geometry that can be readily scaled to practical, device-like sizes.

This research was supported by the WELCOME project "Hybrid Nanostructures as a Stepping Stone towards Efficient Artificial Photosynthesis" funded by the Foundation for Polish Science and EUROCORES project "BOLDCATS" funded by the European Science Foundation. We thank Eckhard Hofmann (University of Bochum, Germany) for providing the PCP complexes. MDT was partially funded by the Purdue Excellence in Science and Engineering Fellowship sponsored by Sandia National Laboratories.

\section{References}

[1] P. Anger, P. Bharadwaj, L. Novotny, Phys. Rev. Lett. 96, 113002 (2006)

[2] M.A. Noginov, G. Zhu, M. Bahoura, C.E. Small, C. Davison, J. Adegoke, V.P. Drachev, P. Nyga, V.M. Shalaev, Phys. Rev. B 74, 184203 (2006).

[3] K. Ray, R. Badugu, J. R. Lakowicz, J. Am. Chem. Soc. 128, 8998 (2006).

[4] W. Zhang, Y. Chen, C. Hu, Y. Zhang, X. Chen, M. Qiu Zhang, J. Mater. Chem. C 1, 1265 (2013).

[5] Ł. Bujak, N. Czechowski, D. Piatkowski, R. Litvin, S. Mackowski, T.H.P. Brotosudarmo, R.J. Cogdell, S. Pichler, W. Heiss, Appl. Phys. Lett. 99, 173701 (2011)

[6] J.B. Nieder, R. Bittl, M. Brecht, Angew. Chem. Int. Ed. 49, 10217 (2010).

[7] P. Nyga, V. P. Drachev, M.D. Thoreson, V. M. Shalaev, Appl. Phys. B 93, 59 (2008).

[8] U.K. Chettiar, P. Nyga, M.D. Thoreson, A.V. Kildishev, V.P. Drachev, V.M. Shalaev, Appl. Phys. B 100, 159 (2010).

[9] V.P. Drachev, M.D. Thoreson, V. Nashine, E.N. Khaliullin, D. BenAmotz, V.J. Davisson, V.M. Shalaev. J. Raman Spectroscopy 36, 648 (2005).

[10] B. Krajnik, T. Schulte, D. Piątkowski, N. Czechowski, E. Hofmann, S. Mackowski, Cent. Eur. J. Phys. 9, 293 (2011). 